\title{
Case Report 107
}

Kathleen P. Heidelberger, M.D., Bertram Schnitzer, M.D., David Tilford, M.D., Andrew K. Poznanski, M.D.*, and Ruth Heyn, M.D.

Departments of Pathology, Pediatrics and Communicable Diseases and the Division of Pediatric Radiology (C.S. Mott Children's Hospital), University of Michigan Medical Center, Ann Arbor, Michigan, USA
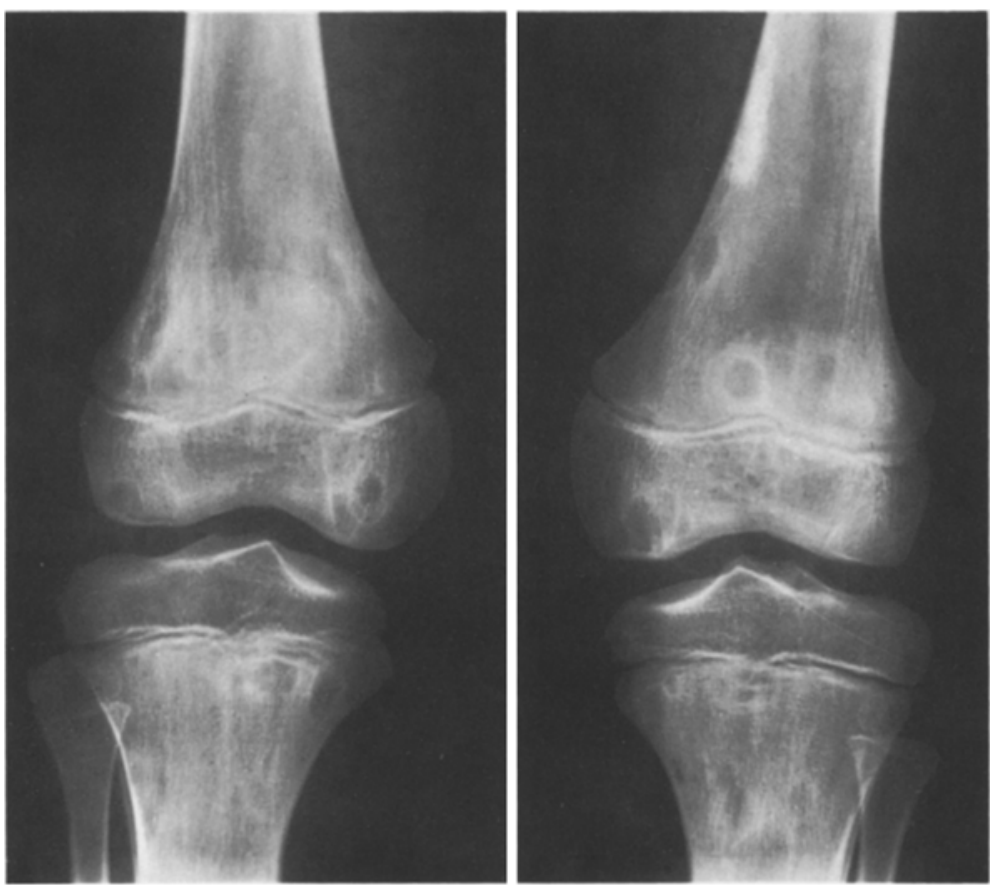

Fig. 1. An anteroposterior roentgenogram of both knees shows numerous lytic defects with sclerotic margins involving the metaphyses and epiphyses of the femora and tibiae. Each patella is also affected
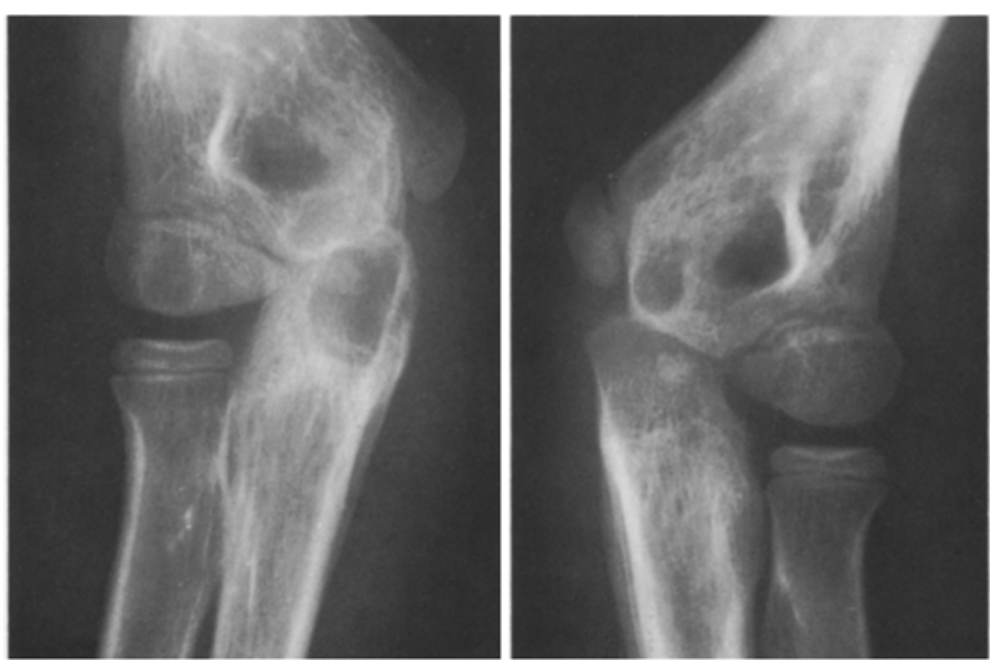

Fig. 2. An anteroposterior roentgenogram of both elbows demonstrates slightly larger but similarly appearing lytic lesions with sclerotic margins in each humerus and ulna. Periosteal reaction is observed around both ulnae

Presented by Dr. Andrew K. Poznanski at the Fifth Annual Meeting of the International Skeletal Society in Boston, Massachusetts, September 4-6, 1978

Address reprint requests to: A.K. Poznanski, M.D., Department of Radiology, The Children's Memorial Hospital, 2300 Children's Plaza, Chicago, IL 60614 USA 


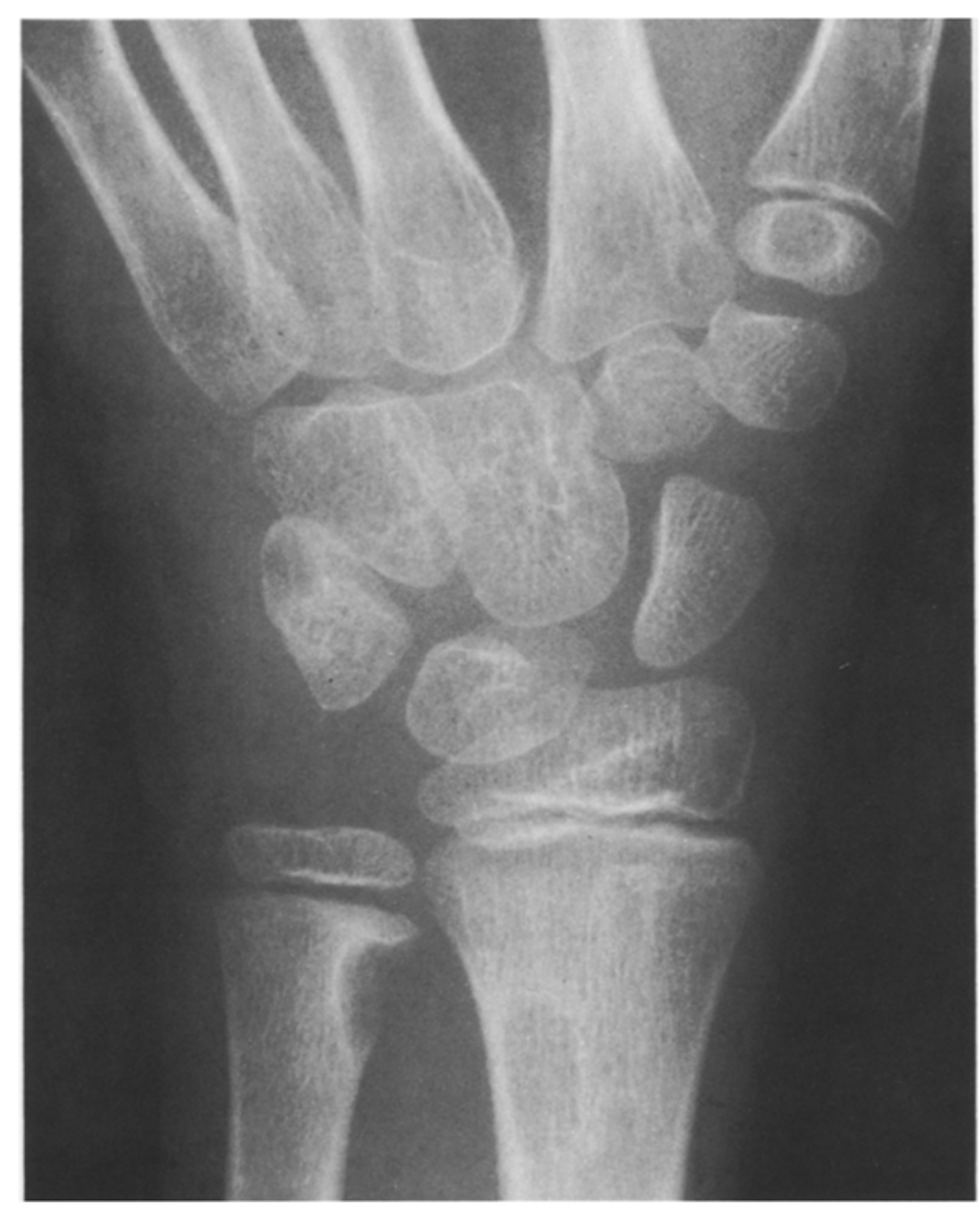

Fig. 3. An anteroposterior roentgenogram of the left wrist demonstrates similar lesions in the ulnar metaphysis, the hamate and the epiphysis of the first metacarpal

\section{History}

This 11-year-old boy presented with a history of pain around both ankles of 5 months duration. The pain was aggravated by walking and running and awakened the child at night. He also complained of transient pains in two fingers and in the left elbow. Four months prior to admission he developed enlarged lymph nodes in the neck, unaccompanied by sore throat, ear infection or fever. He was given oral antibiotics with no effect on the size of the nodes. A 12 pound weight loss ensued. A lymph node biopsy was performed two weeks prior to admission. At that time radiological examination of the ankles showed multiple lytic bone lesions which led to his referral to University Hospital.

On admission to the hospital the patient was noted to be a thin boy in no distress. Enlarged cervical lymph nodes of varying size were palpated bilaterally. Neither hepatosplenomegaly nor skin lesions was present. A $1.5 \mathrm{~cm}$ node was noted in each inguinal region.

The temperature was normal. The hemoglobin was $11.8 \mathrm{gm} / \mathrm{dl}$, platelet count $524,000 / \mathrm{mm}^{3}$ and WBC $10,700 / \mathrm{mm}^{3}$ with 69 segmented neutrophils, 15 bands, 7 lymphocytes, 7 monocytes and 2 eosinophils. The absolute lymphocyte count was $749 \mathrm{~mm}^{3}$. The ESR was $56 \mathrm{~mm} / \mathrm{hr}$. The monospot ${ }^{\mathrm{R}}$ test was negative. EBV titers were not done. Serum calcium, alkaline phosphatase, renal function tests and liver enzymes were normal. The IgG was $1416 \mathrm{mg} / \mathrm{dl}$, IgA $299 \mathrm{mg} / \mathrm{dl}$ and $\operatorname{IgM} 164 \mathrm{mg} / \mathrm{dl}$.

A skeletal survey demonstrated multiple, well-defined, lytic lesions disseminated throughout the skeleton (Figs. 1-3). Radiological examination of the chest, barium swallow and lymphangiography were all normal. An excretory urogram showed the kidneys to be enlarged more than 2 standard deviations above the mean size.

A bone marrow aspirate and biopsy showed increased myelopoiesis and prominent eosinophils. Lymph node and fibular biopsies were performed (Figs. 4-7). 

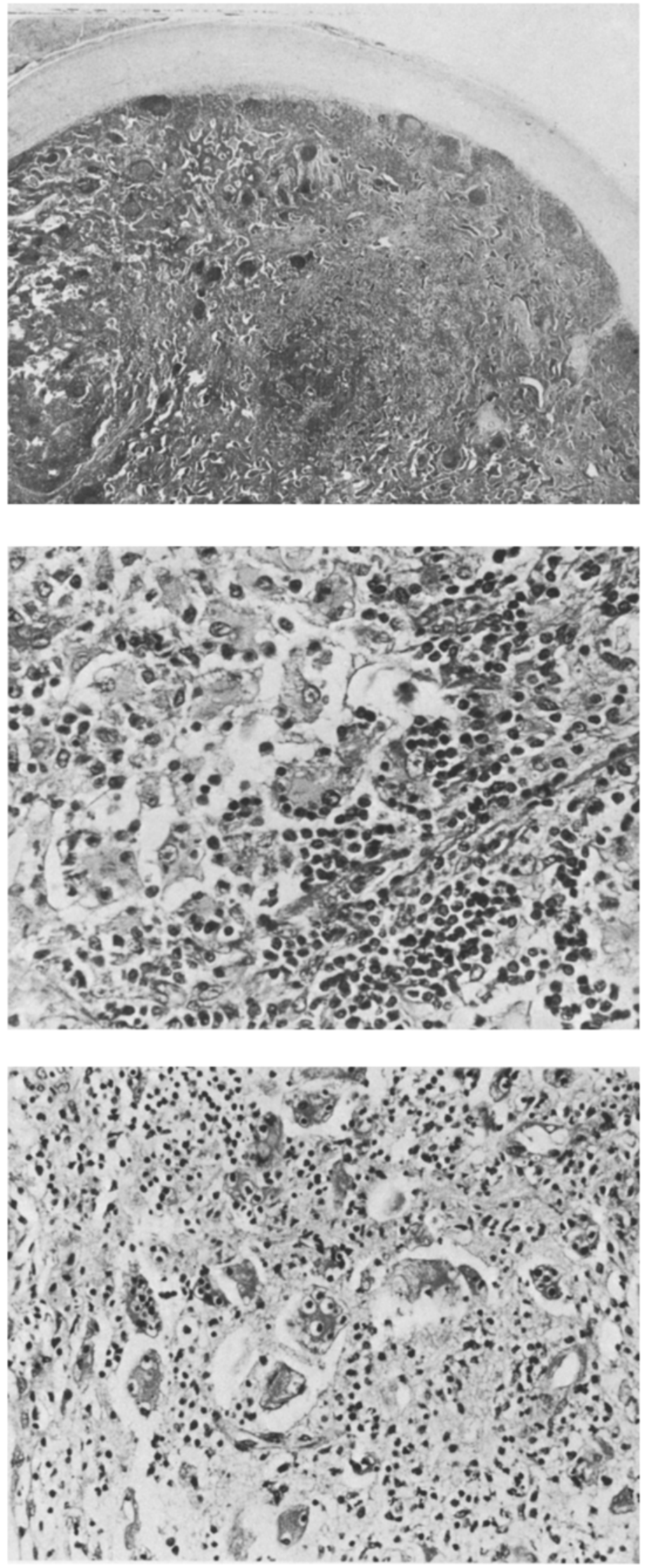

\section{Pathological Studies}

Fig. 4. A photomicrograph obtained after biopsy of a lymph node $(\mathrm{H}$ and $\mathrm{E}$ stain, $\times 12.6)$ shows a thickened fibrous capsule around and subdividing a node

Fig. 5. Another photomicrograph from a biopsy specimen of a lymph node $(\mathrm{H}$ and $\mathrm{E}$ stain $\times 320$ ) shows the peripheral central sinuses to be distended and filled with large, bland-appearing histiocytes, with small nuclei and pale to eosinophilic-staining vacuolated cytoplasm. Some histiocytes demonstrate larger, more vesicular nuclei with prominent nucleoli. Within the cytoplasm of some of the histiocytes, one to several lymphocytes are present, while other histiocytes contain polymorphonuclear leukocytes or an occasional erythrocyte

Fig. 6. A photomicrograph obtained from a biopsy specimen of a skeletal lesion (decalcified material $-\mathrm{H}$ and E stain $\times 205$ ) shows multiple histiocytes of varying sizes containing phagocytosed lymphocytes. Interspersed lymphocytes are present 


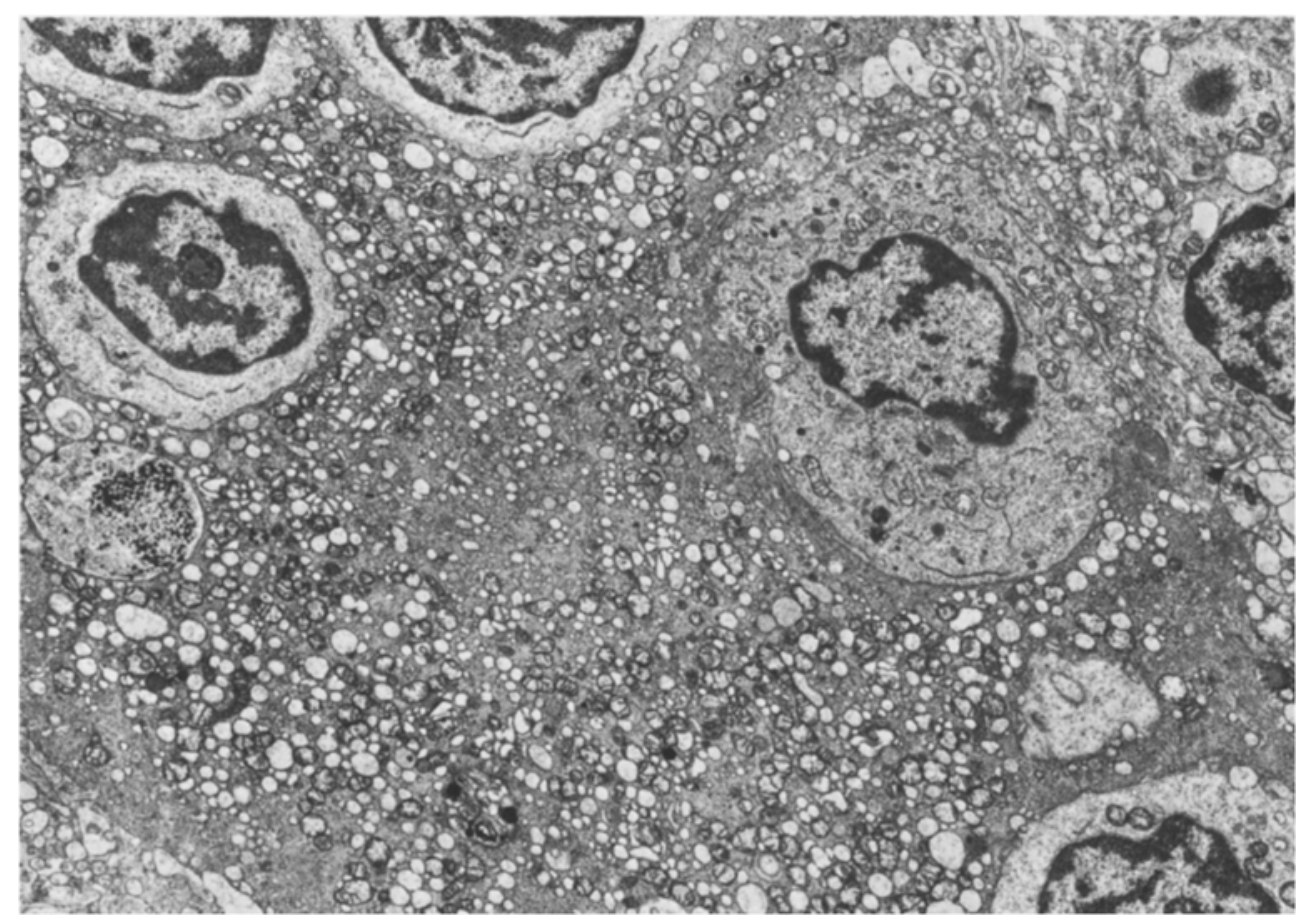

Fig. 7. An electron-micrograph of a section of cytoplasm obtained from a bone biopsy shows histiocytes with irregular nuclei containing a small amount of heterochromatin along the nuclear membrane. One to two nucleoli are noted in some nuclei. The cytoplasm of a histiocyte demonstrated is noted to contain numerous mitochondria, many of which are rod-shaped. Electron-dense lipid bodies are scattered throughout the cytoplasm. Five intact lymphocytes are observed within the cytoplasm of the histiocyte. Langerhans granules were not identified

\section{Diagnosis: Sinus Histiocytosis with Massive Lymphadenopathy and Gross Skeletal Involvement}

The differential diagnosis should include histiocytosis (Hand-Schuller-Christian variant), a disseminated infective process (e.g., tuberculosis), multiple nonossifying fibromatosis and conceivably neoplastic disease (e.g., metastases from neuroblastoma, lymphoma)

In the ten months of follow-up after discharge from the hospital, the child has been asymptomatic except for intermittent skeletal pain. He has gained 10 kilograms and his lymph nodes have decreased in size considerably. Follow-up radiological skeletal surveys have shown partial healing of the lytic lesions.

\section{Discussion}

Sinus histiocytosis with massive lymphadenopathy (SHML) was described as a new entity in 1969 by Rosai and Dorfman. A more detailed description of 34 cases was reported by the same authors in 1972 . Eleven patients had skeletal surveys, but only one was said to have mixed lytic and blastic lesions. No significant bone changes were observed at autopsy in this patient. Further tabulation of 113 cases in 1977 stressed the non-nodal features of the entity. Five cases were reported to have bone involvement, but further details were not given. In 1976 Ramos reported a 3-year-old black girl who had widespread bone involvement with SHML. Osteolytic lesions were present in the diaphyses and metaphyses of long bones and metacarpals. The histological features of a tibial lesion were similar to that observed in the lymph nodes.

The present case constituted a dilemma until the histological studies of the lymph node and bone biopsies clarified the diagnosis. The patient had several features previously described, including lymphadenopathy, lytic lesions of the skeleton, weight loss and lymphopenia. His lymph nodes, however, were not massively enlarged and his skeletal lesions were more extensive than has been reported previously. A recent report by Thawerani et al. stressed 
the point that some cases presenting with extranodal involvement may not have massive lymphadenopathy. These authors also noted that if the first tissue biopsy is extranodal in origin, the histological pattern may be variable and confusing.

The presence of the characteristic histological features described in this patient is necessary to establish the diagnosis. The pathogenesis is unknown and the reason for the relatively infrequent appearance of skeletal lesions is not determined.

The case is important because it documents another cause of well-defined disseminated skeletal lesions with lymphadenopathy in an entity which has not received up to the present time any prominent identification by radiologists, orthopedic clinicians and very likely even pathologists. Although relatively few cases show skeletal lesions, the tabulation in 1977 by Sanchez, Rosai and Dorfman of 113 cases indicates clearly that the entity is more common than appreciated.

\section{References}

1. Ramos, C.V.: Widespread bone involvement in sinus histiocytosis. Arch. Pathol. Lab. Med. 100, 606 (1976)

2. Rosai, J., Dorfman, R.F. : Sinus histiocytosis with massive lymphadenopathy. A newly recognized benign clinicopathological entity. Arch. Pathol. 87, 63 (1969)

3. Rosai, J., Dorfman, R.F. : Sinus histiocytosis with massive lymphadenopathy. A pseudolymphomatous benign disorder. Cancer 30, 1174 (1972)

4. Sanchez, R., Rosai, J., Dorfman, R.F.: Sinus histiocytosis with massive lymphadenopathy: An analysis of 113 cases with special emphasis on its extranodal manifestations (Abstract). Lab. Invest. 36, 349 (1977)

5. Thawerani, H., Sanchez, R.L., Rosai, J., Dorfman, R.F.: The cutaneous manifestations of sinus histiocytosis with massive lymphadenopathy. Arch. Dermatol. 114, 191 (1978) 